\title{
On how to extract breathing rate from PPG signal using wearable devices
}

\author{
A. Fusco ${ }^{1}$, D. Locatelli ${ }^{1}$, F. Onorati ${ }^{2}$, G. C. Durelli ${ }^{1}$,M. D. Santambrogio ${ }^{1}$ \\ ${ }^{1}$ DEIB, Politecnico di Milano, Milan, Italy, ${ }^{2}$ Empatica Inc. \\ Emails: \{alessandra1.fusco, davide3.locatelli\}@mail.polimi.it, \\ fo@empatica.com, \{gianlucacarlo.durelli, marco.santambrogio\}@ polimi.it
}

\begin{abstract}
In this paper, an algorithm for breathing rate extraction from PPG signal is proposed. Two critical aspects have been endorsed during the implementation: i) good performances and ii) low computational complexity. The proposed solution is based on the Empirical Mode Decomposition (EMD) approach and it proves to be robust and accurate even in presence of noisy epochs. It has been validated on two distinct datasets: a)experimental data we have collected using wearables for physiological monitoring and b) recording sessions from PhysioBank MIMIC II Waveform Database. The presented results showed a mean absolute error of $0.0044 \mathrm{~Hz}$, corresponding to 0.26 breaths per minute.
\end{abstract}

\section{INTRODUCTION}

The breathing rate (BR) measurement provides vital information about the patient's health [1]; it is crucial in diagnosing various respiratory diseases and it is potentially useful in numerous clinical and non-clinical settings [2], [3]. Currently, there are numerous methods for monitoring the BR. They are classified as direct method if they are directly related to the amount of air exchanged during the respiration act, or as indirect if they are based on physiological indexes indirectly related to respiration. Even though some of the available approaches have been proved to be effective, they still present considerable disadvantages. For instance, direct methods showed to be uncomfortable due to the indispensable use of a mouthpiece. The mask limits the body movements, affecting the normal respiratory pattern. Among indirect approaches, some devices measure the chest wall movements in order to estimate lung volume variations. These systems are minimally invasive and can be implemented in wearable systems. However, textile substrate is easily affected by movement artifacts during sleeping or activities of daily living [4]. These limitations have induced investigators to estimate BR using optical techniques.

Pulse oximetry is one of the most widely used method for vital signs monitoring. It is based on the optical technique known as photoplethysmography. The key principle is simple: light from a transmitter is scattered and partly absorbed in the tissue. Part of the scattered light emerges through the skin and can be detected by a photosensitive detector; the intensity of detected light is represented as a plethysmogram. Figure 1 shows an example of the PPG waveform, composed of the direct current (DC) and the alternating current (AC) parts. The DC component

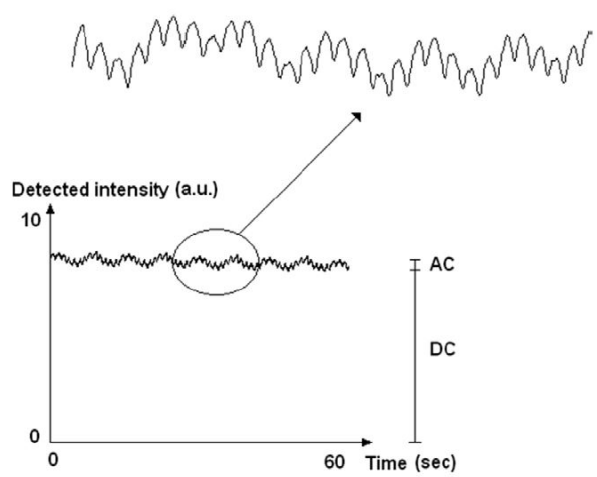

Fig. 1. The direct current $(D C)$ part reflects the optical character of the underlying tissue and the arterial pulsation. At a level above, there are small intensity variations, roughly $1 \%$ to $5 \%$ of the DC level, corresponding to arterial pulsations. a.u. = aubitrary units.

corresponds to the detected transmitted or reflected optical signal, and depends on the structure of the tissue as well as on the average blood volume of both arterial and venous blood. Instead, the AC component shows blood volume variations occurring between the systolic and diastolic phases of the cardiac cycle; the fundamental frequency of the AC component depends on the heart rate and is superimposed onto the DC component.

Since the photoplethysmographic (PPG) signal contains a respiratory component, the BR extraction from PPG signal is a current topic in the scientific community. The small size of the probe, the possibility of continuous monitoring, and few side effects [5] are additional benefits of this method. In the last decade, several approaches addressing PPG-derived BR have been proposed. Despite good performances have been achieved, algorithm complexity and computational requirements have prevented real-time applications [6], [7].

The novel contribution of this research can be summarized as follows:

- good accuracy in estimating BR;

- low computational cost of the algorithm;

- real-time signal processing for wearable applications.

A general review of BR estimators from PPG signal is provided in Section II. Following, the novel approach 
is presented and compared to Madhav's [7], which was the starting point for the development of our method. The algorithm is validated on two distinct datasets, one already proposed in the Literature, and the other we experimentally collected using wearable devices. Experimental results are analysed in order to evaluate algorithm performances.

\section{RELATED WORKS}

Different studies have achieved high levels of accuracy in extracting BR from PPG signal; nevertheless, their computational complexity prevent real-time applications. In contrast, further research projects proposed algorithm for real-time applications but they reported an average error of 3 breaths per minute.

For instance, Leonard et al. [6] proposed a fully automated algorithm for BR extraction from PPG signal. The PPG signal is decomposed three times via Morlet wavelet transform to produce three different breathings signal. The 'best' source for each five (5) minute section is chosen as the one with the lowest absolute error when compared to the reference. Despite this approach revealed a mean absolute error of 0.31 breaths per minute, it is not applicable to real applications when a reference frequency is not available. Furthermore the algorithm complexity and computational difficulty constitute remarkable limits.

In 2013, Karlen at al. [8] suggested a method, known as Smart Fusion, for estimating BR in real-time from the PPG signal. Three respiratory-induced variations (frequency, intensity, and amplitude) are extracted from PPG signal and the frequency content of each parameter is analysed in the frequency domain. Hence, the BR is estimated using an algebraic mean calculation. Despite the light computational cost of the algorithm, the Smart Fusion method revealed an average error of 3 breaths per minute. This error is remarkably high, and it is not considered admissible both for clinical and non-clinical settings.

Within this context, the algorithm presented below was designed as a trade-off between performances and computational cost.

\section{PROPOSED APPROACH}

In 2011, Madhav et al. [7] proposed a method based on the Empirical Mode Decomposition (EMD) for estimating BR from PPG signal. EDM is an adaptive time-frequency data analysis; it has proven to be quite versatile in a broad range of applications for extracting signals from data generated in noisy non-linear and non-stationary processes. The key point is to identify the oscillatory modes at different time scales; thus, the signal is decomposed accordingly into a set of frequency-and-amplitude modulated components, called Intrinsic Mode Functions (IMF). The EMD algorithm can be summarized in the following steps:

1) Find the local maxima, $M_{i}, i=1,2 \ldots$, and the local minima, $m_{k}, k=1,2, \ldots$, in the signal $x(t)$.
2) Compute the corresponding interpolated signals $M(t):=f_{M}\left(M_{i}, t\right)$, and $m(t):=f_{m}\left(m_{k}, t\right)$. These signals are the upper and lower envelopes of the signal.

3) Let $e(t):=(M(t)+m(t)) / 2$.

4) Subtract $e(t)$ from the signal: $x(t):=x(t)-e(t)$.

5) Return to step 1. - stop when $x(t)$ remains nearly unchanged.

6) An IMF, $\phi(t)$, corresponds to the remaining signal $x(t)$;

7) $\phi(t)$ is removed from the signal of the first step: $x(t):=x(t)-\phi(t)$. If $x(t)$ has more than one extremum (neither a constant nor a trend), the iterative procedure is repeated.

The stopping criterion (Step 5) is merely empirical and it could affect the performance of the method. For instance, criteria based on number of iterations do not guarantee a scale independent stop.

In the preliminary phase of our study, a smoothing filter is applied in order to remove unwanted components from the PPG signal facilitating the application of the EMD method.



Fig. 2. The PSD of filtered PPG signal obtained via Fourier transform.

When decomposing the PPG signal via EMD, we observed that two principal components can fully describe the PPG signal dynamic (see Figure 2): the component corresponding to the highest frequency is an estimate of the Heart Rate (HR), while the component corresponding to the lowest frequency is an estimate of the BR. Thus, in our approach the EMD is stopped at the first loop, in correspondence to the mean envelope calculation (Figure 3). The novel formulation certainly reduces the algorithm complexity and the computational difficulty. Lastly, the mean envelope is used to estimate BR using frequency analysis. The power spectral density (PSD) of the respiratory component is used to evaluate the dominant frequency peak.

In order to assess the accuracy of the proposed approach and to investigate the feasibility of extracting BR using wearable devices, two distinct datasets have been evaluated: data we have experimentally collected and recording sessions from PhysioBank MIMIC II Waveform Database [9]. All sessions of both datasets include PPG and simultaneously 




Fig. 3. The mean envelope extraction from PPG signal.

recorded respiration signal. The latter is used as "ground truth" in the validation of the algorithm. Sixty (60) oneminute epochs, not affected by missing data nor signal saturation artifacts, were selected from different sessions of each dataset.

Concerning the database we collected experimentally using wearable devices, physiological signals are acquired continuously from adult subjects without known respiratory diseases. In order to minimize movement artifact noise, sleeping sessions are analysed. PPG signal is obtained through a commercial smartwatch for physiological monitoring [10]. Whereas, the respirogram is recorded by means of a wearable chest band. The band detects chest expansion and contraction, showing relative thoracic wall movements.

As regards the public database, the MIMIC II (Multiparameter Intelligent Monitoring in Intensive Care) database is part of the PhysioNet archive of biomedical signal databases. The archive contains physiologic signals captured from patient monitors, and comprehensive clinical data obtained from hospital medical information systems, for over ten thousands Intensive Care Unit (ICU) patients.

\section{EXPERIMENTAL RESULTS AND DISCUSSIONS}

The experimental validation of the approach here presented is illustrated in the following section. Standard statistical tests are applied in order to compare the effectiveness of the novel method. The procedure is repeated for both the datasets.

\section{A. Results on Experimental Dataset}

With regard to our dataset, the EMD-based method reveals good performances in estimating BR from PPG signal. The Mean Absolute Error (MAE) is $0.0027 \mathrm{~Hz}$, corresponding to 0.19 breaths per minute; the Spearman's correlation coefficient $\left(\rho_{s}\right)$ is 0.962 .

Figure 4 shows the Bland-Altman plot of the difference between the ground truth and the EMD-based BR estimates;

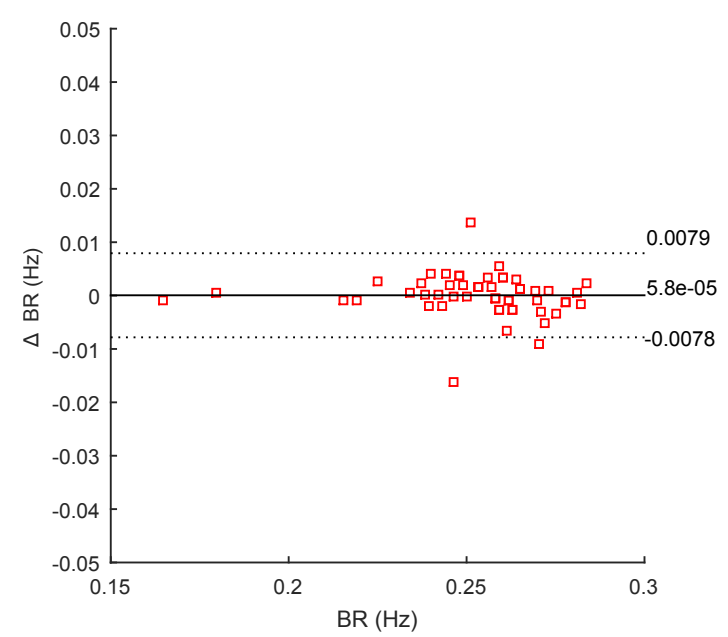

Fig. 4. Bland-Altman plot showing the distribution of the difference between the BR estimated with the EMD-based method on experimental data and the ground truth.

despite the errors in the estimation are relatively small, the presence of outliers affects the results. This situation is reflected in the correlation between the ground truth values and our algorithm estimates.

\section{B. Results on Physiobank Archive}

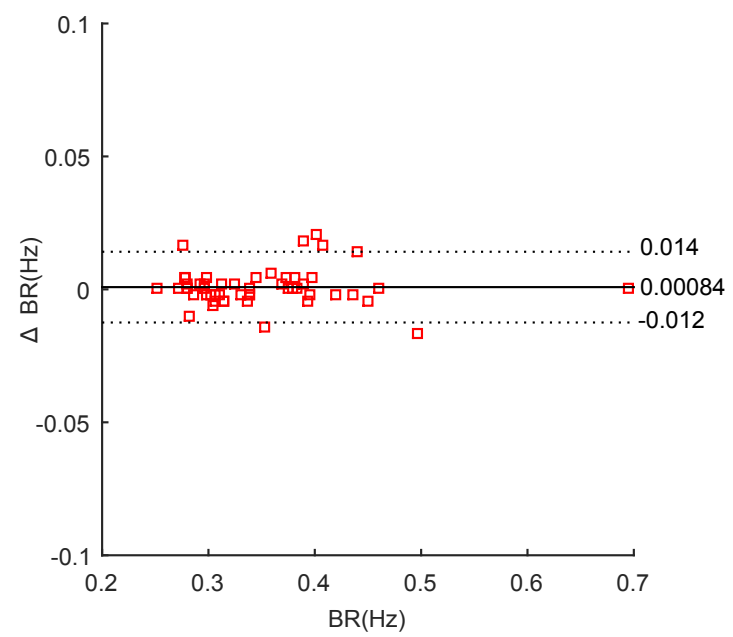

Fig. 5. Bland-Altman plot showing the distribution of the difference between the BR estimated with the EMD-based method on Physiobank data and the ground truth.

With regard to the PhysioBank archive, the here presented approach shows high performances in estimating BR from the PPG signal. The Mean Absolute Error (MAE) is $0.0044 \mathrm{~Hz}$, corresponding to 0.26 breaths per minute; the Spearman's correlation coefficient $\left(\rho_{s}\right)$ is 0.991 .

Figure 5 shows the Bland-Altman plot of the difference between the ground truth and the EMD-based BR estimates; it can be noted that the errors in the estimation are relatively 
small. The effectiveness of the algorithm is also confirmed by the strong correlation between the ground truth values and our algorithm estimates. Hence, it can be concluded that no bias nor offset is caused by the algorithm.

Although Madhav et al. method seems to outperform our results on the same database, they analysed only five (5) one-minute neat epochs. Moreover, the complex and computationally heavy iterative procedure employed would prevent real-time applications. By contrast, our simplified approach proves to be robust and accurate on a more extended dataset even when processing noisy epochs or analysing recordings with abnormal BR (i.e. $0.7 \mathrm{~Hz}$ ).

\section{Discussions}

TABLE I

MEAN ABSOLUTE ERRORS (MAES) AND SPEARMAN'S CORRELATION COEFFICIENT $\left(\rho_{S}\right)$ FOR EACH DATASETS.

\begin{tabular}{|l|c|c|}
\hline Method & MAE (Hz) & $\rho_{s}$ \\
\hline Experimental Data & 0.0027 & 0.962 \\
\hline MIMIC II Data & 0.0040 & 0.991 \\
\hline
\end{tabular}

The EMD-based approach shows good performances in estimating BR from PPG signal. Comparing the results on the two distinct datasets (Table I) it is noticeable how the correlation coefficient resulting from the experimental dataset is slightly inferior to that resulting from PhysioBank data.
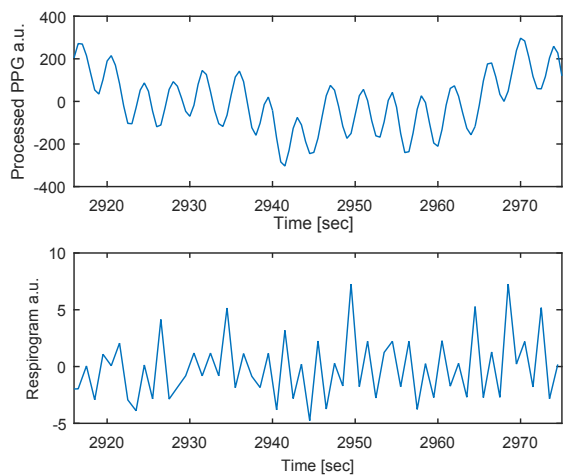

Fig. 6. The graph compares the PPG signal processed with the envelope approach with the refererence noisy respirogram.

After having analysed the recordings, we noticed that outliers originate mainly in movement artifacts. The adherence of the chest band is not assured: during sleeping sessions, the body movements could alter the results and thus the respiratory signal used as "ground truth". Even though the BR estimated from PPG signal seems reasonable, the reference signal is corrupted (Figure 6); as a result, the correlation coefficient is appreciably reduced $(0.962$ versus $0.991)$.

The results confirmed that reliability of methods currently used for respiratory monitoring is not guaranteed. Despite the chest band constitutes one of the most widely used techniques for respiratory monitoring, it proved to be unreliable in ordinary daily life. Since the key principle is based on chest expansion, the measured respiratory signal is extremely affected by movements artifacts. Furthermore, the chest band is generally judged uncomfortable.

On the contrary, the wristband has revealed to be a more accurate measurement device: it is feasible for daily activities and it is easier to use than the chest band.

\section{CONCLUSIONS}

In this contribution, we showed that it is possible to track BR from PPG signal with high accuracy at a low computational cost. Despite the relatively simple structure of the algorithm, the results indicate a strong correlation with the ground truth. Analysis of the PPG signal offers an alternative way of monitoring $\mathrm{BR}$; this indirect estimation can be extremely useful in situations when a continuous monitoring is required. Non-invasiveness, the possibility of mobile monitoring and a few negative side effects are attractive attributes of photoplethysmography.

In particular, in the area of wearable devices, fast and robust algorithms are crucial requirements for real-time applications.

\section{ACKNOWLEDGMENT}

This work was developed in and supported by Empatica Inc.

\section{REFERENCES}

[1] Yuan, George, Nicole A. Drost, R. Andrew McIvor. "Respiratory rate and breathing pattern." McMaster University Medical Journal 10.1 (2013): 23-25

[2] Fieselmann JF, Hendryx MS, Helms CM, et al. Respiratory rate predicts cardiopulmonary arrest for internal medicine patients. J Gen Intern Med 8 (1993): 354-360.

[3] Subbe, C. P., Davies, R. G., Williams, E., Rutherford, P., Gemmell, L. "Effect of introducing the Modified Early Warning score on clinical outcomes, cardio-pulmonary arrests and intensive care utilisation in acute medical admissions". Anaesthesia 58 (2003): 797-802.

[4] Sepp, V. P., Visnen, J., Lahtinen, O., Hyttinen, J. "Assessment of breathing parameters during running with a wearable bioimpedance device." 4th European Conference of the International Federation for Medical and Biological Engineering. Springer Berlin Heidelberg, 2009.

[5] Sinex, James E. "Pulse oximetry: principles and limitations." The American journal of emergency medicine 17.1 (1999): 59-66.

[6] Leonard, P. A., Douglas, J. G., Grubb, N. R., Clifton, D., Addison, P. S., Watson, J. N. "A fully automated algorithm for the determination of respiratory rate from the photoplethysmogram" Journal of Clinical Monitoring and Computing, 20.1 (2006): 33-36.

[7] Madhav, K. V., Ram, M. R., Krishna, E. H., Komalla, N. R., Reddy, K. A. "Estimation of respiration rate from ECG, BP and PPG signals using empirical mode decomposition". In Instrumentation and Measurement Technology Conference (I2MTC), 2011 IEEE (pp. 1-4). IEEE.

[8] Karlen, W., Raman, S., Ansermino, J. M., Dumont, G. A. "Multiparameter respiratory rate estimation from the photoplethysmogram." Biomedical Engineering, IEEE Transactions on 60.7 (2013): 1946 1953.

[9] Goldberger AL, Amaral LAN, Glass L, Hausdorff JM, Ivanov PCh, Mark RG, Mietus JE, Moody GB, Peng C-K, Stanley HE. "PhysioBank, PhysioToolkit, and PhysioNet: Components of a New Research Resource for Complex Physiologic Signals." Circulation 101(23), (2000): e215-e220.

[10] E4 Wristband developed by Empatica Inc., Boston, Massachusetts, USA (https://www.empatica.com/e4-wristband) 